\title{
13. PALEOMAGNETISM OF SOME LEG 27 SEDIMENT CORES
}

\author{
Richard D. Jarrard, Scripps Institution of Oceanography, La Jolla, California
}

\section{METHODS}

Cretaceous cores from Site $260 \quad\left(16^{\circ} 8.67^{\prime} \mathrm{S}\right.$, $\left.110^{\circ} 17.92^{\prime} \mathrm{E}\right)$; Site $261\left(12^{\circ} 56.83^{\prime} \mathrm{S}, 117^{\circ} 53.56^{\prime} \mathrm{E}\right)$; and Site $263\left(23^{\circ} 19.43^{\prime} \mathrm{S}, 110^{\circ} 57.81^{\prime} \mathrm{E}\right)$ were sampled for paleomagnetic measurements. Except for the upper cores from Site 260, which were sampled by pressing the 5.8-cc rectangular sample boxes into the split core face, all samples were cut on a diamond saw. The cores consist of cylindrical pieces of undeformed claystone and siltstone from a few centimeters to tens of centimeters in length. Drilling caused the pieces to rotate horizontally by an unknown amount. Rather than a uniform sampling interval, several adjacent samples from the same piece were often taken to minimize breakup of core material, expedite sampling, and enable comparison of declination data from samples known to have undergone no horizontal rotation relative to each other.

Table 1 shows the directions of remanent magnetization and intensities of the samples after 50-oe ac demagnetization. Samples were measured at $5 \mathrm{~Hz}$ on a Schonstedt magnetometer. An asterisk in Column 1 indicates that the Schonstedt magnetometer and ac demagnetization apparatus at Massachusetts Institute of Technology were used (see Green and Brecher [this volume] for detailed discussion of these measurements). All other samples were measured using the Schonstedt magnetometer and ac demagnetization apparatus at the University of California at Santa Barbara. Sample Interval in Table 1 is the distance in centimeters from the top of the 2-cm-wide sample to the top of the section. Inclinations (Incl) are given with respect to the present horizontal, positive downward, assuming vertical drill holes; negative inclinations indicate normal polarities for these southern hemisphere cores. Declinations (Decl) are relative only to the split face of the core.

\section{RELIABILITY OF MEASUREMENTS}

$\mathrm{MM}$ and $\mathrm{A}$ are measures of the consistency of replicate measurements on a single sample (Doell and Cox, 1965). Values of MM greater than about $30 \%$ or of A greater than about $5^{\circ}$ indicate that the intensity or direction of magnetization, respectively, are probably unreliable, generally because the sample intensity is near the noise level of the magnetometer or because of instability of sample magnetization.

Table 2 shows results of ac demagnetization series on four samples. Little change in direction of magnetization upon demagnetization is apparent, indicating that postdepositional viscous components of magnetization are small. Cumulative intensity changes for these samples (Figure 1) show that a majority of the magnetization resides in domains of high coercive force.
Fifty oersted was chosen as the minimum demagnetizing field needed to remove viscous components. Histograms of 50-oe inclinations are generally considerably steeper than the geocentric axial dipole inclinations for the present latitudes of the sites. If a significant viscous component is present in these 50 -oe measurements,lower average inclinations and higher average intensities should result for normally polarized samples than for reversely magnetized samples. Too few reversely magnetized samples are available from Site 260 , Site 261, or from Site 263 samples run at UCSB, to assess the presence of viscous components in these 50 -oe measurements. A rank sum test of 50 oe measurements of Site 263 sediments run a MIT demonstrates that normally polarized samples do not have significantly lower inclinations or higher intensities than reversed samples: consequently, no significant viscous magnetization is present in these measurements.

As implied by Figure 2 and demonstrated by a rank sum test, samples measured at MIT have significantly higher inclinations, by about $5^{\circ}$, than samples of about the same age from the same site measured at UCSB. The median to-oe inclination for Site 263 samples measured at UCSB is $56.6^{\circ}\left(95 \%\right.$ confidence limits $\left.54.9^{\circ}-59.2^{\circ}\right)$. The median inclination for MIT measurements is $63.2^{\circ}$ $\left(95 \%\right.$ confidence limits $\left.60.6^{\circ}-64.8^{\circ}\right)$. The cause of this discrepancy is probably an anhysteretic component in the UCSB demagnetization apparatus.

\section{REVERSAL STRATIGRAPHY}

Magnetic anomalies at sea show the presence of a wide quiet zone beyond anomaly 33 . The quiet zone, probably representing a long interval of almost exclusively normal polarity (Helsley and Steiner, 1969), has been approximately dated by Deep Sea Drilling as Campanian (Sclater et al., 1974) to Barremian (Sclater, personal communication), in fairly good agreement with polarities of continental rocks (Irving, 1973; Pechersky and Khramov, 1973).

Figures 3-5 show inclinations as a function of depth at Sites 260,261 , and 263 , respectively.

The Site 260 intervals sampled are dominantly of normal polarity, with a clear reversal in Core 17 and with short intervals of very low inclinations and consequently indeterminate polarity in parts of Cores 12 and 18. No paleontological zonal assignment was possible for Core 7; Cores 8-11 are middle to upper Albian and Cores 12-18 are middle to lower Albian. Previous paleomagnetic studies of rocks of Albian age have shown either entirely normal polarity (Helsley and Steiner, 
TABLE 1

Sample Measurements

After 50-oe Demagnetization

\begin{tabular}{|c|c|c|c|c|c|}
\hline $\begin{array}{l}\text { Core-Section } \\
\text { Interval }(\mathrm{cm})\end{array}$ & $\begin{array}{l}\text { Incl. } \\
(\circ)\end{array}$ & $\begin{array}{l}\text { Decl. } \\
(\circ)\end{array}$ & A & $\begin{array}{l}\text { Intensity } \\
\times 10^{-6} \frac{\mathrm{emu}}{\mathrm{cc}}\end{array}$ & $\begin{array}{l}\mathrm{MM} \\
(\%)\end{array}$ \\
\hline \multicolumn{6}{|l|}{ SITE 260} \\
\hline $7-2,106$ & -46.7 & 63.5 & 1.7 & 35.1 & 31 \\
\hline $7-2,140$ & -57.0 & 288.1 & 9.0 & 43.7 & 48 \\
\hline $7-3,5$ & -45.1 & 158.9 & 0.6 & 68.1 & 11 \\
\hline $7-3,67$ & -39.8 & 201.0 & 1.5 & 101 & 14 \\
\hline $7-3,110$ & -32.4 & 101.5 & 0.2 & 78.1 & 19 \\
\hline $7-3,138$ & -61.7 & 112.0 & 5.4 & 12.8 & 27 \\
\hline $7-4,6$ & -27.0 & 25.0 & 0.1 & 83.5 & 28 \\
\hline $7-4,25$ & -32.5 & 154.5 & 0.1 & 95.1 & 7 \\
\hline $8-5,94$ & -39.4 & 350.1 & 1.7 & 83.6 & 16 \\
\hline $8-5,110$ & -28.8 & 98.0 & 1.7 & 92.8 & 21 \\
\hline $8-5,139$ & -44.5 & 281.3 & 0.4 & 74.6 & 5 \\
\hline $8-6,8$ & -50.3 & 359.2 & 1.4 & 54.2 & 12 \\
\hline $8-6,50$ & -23.1 & 36.5 & 1.9 & 71.7 & 9 \\
\hline $8-6,92$ & -41.5 & 31.7 & 0.9 & 94.4 & 30 \\
\hline $8-6,100$ & -67.6 & 209.8 & 0.0 & 47.2 & 19 \\
\hline $8-6,142$ & -33.6 & 75.9 & 3.7 & 98.0 & 17 \\
\hline $10-1,22$ & -46.9 & 7.3 & 1.4 & 37.4 & 12 \\
\hline $10-1,68$ & -35.3 & 128.1 & 0.5 & 55.5 & 8 \\
\hline $10-1,109$ & -47.2 & 22.4 & 0.0 & 32.2 & 14 \\
\hline $10-1,134$ & -66.9 & 184.1 & 2.2 & 13.1 & 32 \\
\hline $10-2,3$ & -48.9 & 333.5 & 1.1 & 21.0 & 8 \\
\hline $10-2,41$ & -46.3 & 312.0 & 0.4 & 25.7 & 7 \\
\hline $10-2,64$ & -35.6 & 9.8 & 1.1 & 43.0 & 10 \\
\hline $10-2,83$ & -79.4 & 16.7 & 5.1 & 14.6 & 28 \\
\hline $10-2,116$ & -34.6 & 329.2 & 2.0 & 57.7 & 6 \\
\hline $10-2,144$ & -44.7 & 289.6 & 0.4 & 76.5 & 6 \\
\hline 11-1, 71 & -44.2 & 118.2 & 0.4 & 42.4 & 9 \\
\hline $11-1,80$ & -39.4 & 138.1 & 2.7 & 28.6 & 30 \\
\hline $11-1,94$ & -44.5 & 93.9 & 0.0 & 53.2 & 12 \\
\hline $11-1,102$ & -36.6 & 151.0 & 0.4 & 57.1 & 8 \\
\hline $11-1,121$ & -41.4 & 115.0 & 0.1 & 57.1 & 6 \\
\hline 123 & -25.1 & 104.2 & 0.6 & 85.2 & 53 \\
\hline $12-1,0$ & -33.5 & 14.8 & 0.1 & 104 & 7 \\
\hline 2 & -32.7 & 17.3 & 0.7 & 114 & 5 \\
\hline 4 & -35.1 & 19.0 & 0.9 & 104 & 7 \\
\hline $12-1,50$ & -27.2 & 36.9 & 1.0 & 17.4 & 8 \\
\hline 52 & -27.6 & 47.7 & 0.5 & 16.3 & 6 \\
\hline 54 & -29.1 & 42.5 & 1.2 & 18.4 & 3 \\
\hline $12-1,111$ & -6.0 & 47.0 & 1.4 & 16.3 & 3 \\
\hline 113 & 3.4 & 48.5 & 0.7 & 14.8 & 5 \\
\hline $12-1,125$ & 0.3 & 82.0 & 0.3 & 16.7 & 5 \\
\hline 127 & 1.6 & 84.9 & 0.0 & 29.1 & 21 \\
\hline $12-2,92$ & -45.6 & 187.4 & 0.0 & 47.0 & 7 \\
\hline 94 & -50.6 & 187.4 & 0.8 & 47.3 & 4 \\
\hline 96 & -49.1 & 182.4 & 0.1 & 45.8 & 7 \\
\hline 98 & -48.4 & 182.0 & 1.3 & 36.5 & 9 \\
\hline $12-2,145$ & -43.6 & 39.5 & 0.6 & 57.5 & 8 \\
\hline 147 & -.53 .1 & 38.8 & 0.3 & 58.8 & 6 \\
\hline 149 & -47.9 & 30.2 & 0.6 & 62.1 & 14 \\
\hline $14-1,69$ & -46.2 & 250.9 & 0.9 & 9.79 & 5 \\
\hline $14-1,76$ & -39.2 & 71.6 & 0.4 & 14.0 & 9 \\
\hline $\begin{array}{r}78 \\
15-3 \cdot 11^{a}\end{array}$ & -34.2 & 69.8 & 0.9 & 13.1 & 7 \\
\hline 13 & -77.6 & 62.1 & 1.0 & 1.96 & 9 \\
\hline 15 & -51.6 & 1.7 & 3.1 & 1.34 & 28 \\
\hline $15-3,102$ & -35.9 & 23.4 & 1.1 & 9.81 & 12 \\
\hline 104 & -51.3 & 178.2 & 0.3 & 1.25 & 14 \\
\hline 106 & -66.2 & 173.4 & 11.5 & 1.10 & 20 \\
\hline $15-4,21$ & -64.0 & 286.5 & 0.1 & 3.30 & 11 \\
\hline 25 & -53.0 & 295.3 & 1.2 & 3.23 & 18 \\
\hline $16-1,89$ & -49.1 & 270.1 & 1.7 & 19.5 & 5 \\
\hline 91 & -55.2 & 269.5 & 0.4 & 23.2 & 11 \\
\hline 93 & -60.9 & 259.0 & 0.4 & 5.4 & 10 \\
\hline
\end{tabular}

TABLE 1 - Continued

\begin{tabular}{crrrcr}
\hline $\begin{array}{c}\text { Core-Section } \\
\text { Interval }(\mathrm{cm})\end{array}$ & $\begin{array}{c}\text { Incl. } \\
\left({ }^{\circ}\right)\end{array}$ & $\begin{array}{c}\text { Decl. } \\
\left({ }^{\circ}\right)\end{array}$ & $\mathrm{A}$ & $\begin{array}{c}\text { Intensity } \\
\times 10^{-6} \frac{\mathrm{emu}}{\mathrm{cc}}\end{array}$ & $\begin{array}{r}\text { MM } \\
(\%)\end{array}$ \\
\hline $16-1,95$ & -56.9 & 270.8 & 1.6 & 2.39 & 9 \\
$17-1,81$ & -46.1 & 51.8 & 1.0 & 12.1 & 25 \\
83 & -53.8 & 62.4 & 1.3 & 13.6 & 9 \\
$17-1,121$ & 70.4 & 334.8 & 0.0 & 34.0 & 7 \\
123 & 52.2 & 302.6 & 8.1 & 86.9 & 45 \\
$17-1,138$ & 57.0 & 11.4 & 2.5 & 22.7 & 5 \\
140 & 42.1 & 19.8 & 1.8 & 26.5 & 7 \\
$18-1,131$ & -60.8 & 256.7 & 0.2 & 76.1 & 6 \\
133 & 9.3 & 203.6 & 1.1 & 67.1 & 9 \\
$18-1,142$ & -66.1 & 238.2 & 0.3 & 59.4 & 4 \\
144 & -63.6 & 236.9 & 0.4 & 63.5 & 9
\end{tabular}

SITE 261

$\begin{array}{rrrrcr}19-3,17 & -46.7 & 221.6 & 0.5 & 96.9 & 6 \\ 19 & -39.8 & 228.2 & 0.2 & 100 & 3 \\ 19-3,126 & -47.1 & 182.6 & 1.3 & 161 & 5 \\ 128 & -52.9 & 186.1 & 1.3 & 147 & 5 \\ 20-1,142 & -54.8 & 18.8 & 0.2 & 219 & 6 \\ 144 & -57.2 & 16.3 & 0.1 & 156 & 20 \\ 21-1,87 & -55.7 & 122.6 & 0.2 & 184 & 7 \\ 89 & -55.1 & 122.2 & 0.2 & 184 & 10 \\ 22-3,15 & -54.9 & 50.3 & 0.2 & 133 & 7 \\ 17 & -49.9 & 55.2 & 0.4 & 150 & 6 \\ 22-3,38 & -49.4 & 216.3 & 0.0 & 42.3 & 5 \\ 22-3,126 & -58.5 & 217.4 & 0.2 & 85.5 & 6 \\ 128 & -57.3 & 212.7 & 0.1 & 89.8 & 4 \\ 23-3,40 & -56.1 & 230.3 & 0.1 & 43.6 & 4 \\ 42 & -40.0 & 244.4 & 0.7 & 41.0 & 9 \\ 23-3,88 & -56.7 & 235.1 & 0.3 & 29.4 & 6 \\ 90 & -58.6 & 223.9 & 0.4 & 26.5 & 6 \\ 92 & -56.2 & 243.5 & 0.0 & 41.5 & 5 \\ 31-2,17 & -51.8 & 191.5 & 0.0 & 34.2 & 8 \\ 19 & -51.4 & 185.8 & 0.2 & 42.7 & 7 \\ 31-2,79 & -30.5 & 106.0 & 3.0 & 2.81 & 34 \\ 81^{\mathrm{a}} & & & & & \\ 31-3,58 & -48.7 & 139.3 & 0.1 & 63.5 & 6 \\ 60 & -47.8 & 142.0 & 0.8 & 46.6 & 25 \\ 62 & -50.0 & 146.6 & 0.5 & 43.0 & 5 \\ 31-4,60 & -63.9 & 155.0 & 1.3 & 3.81 & 8 \\ 62 & -56.7 & 153.5 & 3.1 & 6.54 & 4 \\ 32-1,145 & -37.1 & 4.1 & 3.2 & 6.66 & 23 \\ 32-2,5 & -40.3 & 28.5 & 0.7 & 6.69 & 12 \\ 32-2,93 & -41.6 & 49.8 & 0.0 & 58.6 & 6 \\ 32-3,147 & -48.7 & 262.3 & 0.0 & 18.0 & 18 \\ 149 & -30.9 & 289.4 & 35.8 & 18.9 & 19\end{array}$

SITE 263

\begin{tabular}{rrrrrr}
$17-1,15$ & -55.4 & 272.7 & 1.2 & 1.33 & 3 \\
17 & -55.7 & 282.0 & 0.6 & 1.32 & 10 \\
$17-2,53$ & -59.2 & 59.4 & 0.9 & 1.56 & 8 \\
55 & -60.7 & 62.0 & 0.3 & 1.56 & 8 \\
$17-3,104$ & -59.9 & 193.5 & 1.7 & 1.06 & 8 \\
106 & -55.2 & 195.9 & 1.1 & 1.05 & 5 \\
$17-5,35$ & -54.9 & 115.1 & 0.4 & 1.27 & 7 \\
37 & -57.0 & 115.7 & 1.1 & 1.36 & 8 \\
$17-6,143$ & -59.6 & 100.9 & 0.3 & 1.23 & 9 \\
145 & -61.5 & 100.3 & 0.8 & 1.19 & 10 \\
$18-1,12$ & -50.8 & 221.8 & 1.0 & 1.19 & 14 \\
14 & -62.6 & 208.1 & 0.3 & 0.94 & 14 \\
$18-2,147$ & -57.7 & 123.8 & 3.9 & 1.06 & 14 \\
149 & -57.3 & 123.9 & 0.7 & 1.03 & 11 \\
$18-4,34$ & -43.3 & 320.3 & 1.3 & 0.54 & 15 \\
36 & -53.2 & 323.7 & 1.4 & 0.56 & 17 \\
$18-5,119$ & -44.3 & 164.2 & 3.7 & 0.56 & 8 \\
121 & -48.2 & 163.6 & 0.0 & 0.55 & 10 \\
123 & -49.1 & 164.0 & 4.1 & 0.58 & 11 \\
\hline
\end{tabular}


TABLE 1 - Continued

\begin{tabular}{|c|c|c|c|c|c|}
\hline $\begin{array}{l}\text { Core-Section } \\
\text { Interval }(\mathrm{cm})\end{array}$ & $\begin{array}{l}\text { Incl. } \\
(0)\end{array}$ & $\begin{array}{c}\text { Decl. } \\
(\circ)\end{array}$ & A & $\begin{array}{l}\text { Intensity } \\
\times 10^{-6} \frac{\mathrm{emu}}{\mathrm{cc}}\end{array}$ & $\begin{array}{l}\text { MM } \\
(\%)\end{array}$ \\
\hline $19-1,124$ & -56.8 & 116.4 & 0.5 & 0.86 & 7 \\
\hline 126 & -52.0 & 127.9 & 0.7 & 0.87 & 6 \\
\hline $19-2,146$ & -50.2 & 172.3 & 0.6 & 0.82 & 9 \\
\hline 148 & -51.7 & 180.5 & 2.3 & 0.80 & 5 \\
\hline 150 & -46.3 & 173.4 & 3.5 & 0.67 & 17 \\
\hline $19-4,16$ & -65.1 & 28.7 & 0.9 & 0.79 & 14 \\
\hline 18 & -68.3 & 41.6 & 0.3 & 0.86 & 10 \\
\hline $19-5,145$ & -56.6 & 127.7 & 0.2 & 0.93 & 11 \\
\hline 147 & -52.4 & 128.1 & 0.5 & 0.95 & 9 \\
\hline 149 & -50.2 & 140.1 & 0.7 & 0.99 & 8 \\
\hline $20-1,100 *$ & -65.1 & 83.9 & & 0.35 & \\
\hline $102 *$ & -67.9 & 92.1 & & 0.28 & \\
\hline $20-3,26 *$ & 64.8 & 233.3 & & 0.70 & \\
\hline $28^{*}$ & 65.9 & 240.8 & & 0.69 & \\
\hline 30 & 61.3 & 253.3 & 0.1 & 0.88 & 7 \\
\hline 32 & 68.2 & 245.3 & 0.8 & 0.78 & 10 \\
\hline $20-5,0$ & -69.5 & 160.9 & 0.0 & 1.12 & 9 \\
\hline 2 & -61.3 & 163.2 & 0.1 & 1.00 & 13 \\
\hline 4 & -61.6 & 172.9 & 1.2 & 0.87 & 10 \\
\hline 6 & -68.6 & 173.0 & 2.4 & 0.79 & 9 \\
\hline $20-6,144$ & -64.9 & 254.2 & 0.0 & 0.84 & 12 \\
\hline 146 & -67.2 & 258.7 & 0.2 & 0.81 & 12 \\
\hline 148 & -59.6 & 258.0 & 1.8 & 0.83 & 9 \\
\hline 150 & -65.2 & 268.5 & 1.6 & 0.71 & 11 \\
\hline $21-2,0^{*}$ & -68.6 & 12.8 & & 0.76 & \\
\hline 2 & -59.0 & 12.1 & 2.3 & 0.87 & 12 \\
\hline $4 *$ & -68.6 & 32.6 & & 0.59 & \\
\hline $21-4,6 *$ & 55.1 & 12.3 & & 0.54 & \\
\hline $8^{*}$ & 62.8 & 12.6 & & 0.52 & \\
\hline 10 & 41.2 & 15.6 & 5.2 & 0.72 & 18 \\
\hline $12 *$ & 64.5 & 11.9 & & 0.60 & \\
\hline $14 *$ & 64.2 & 356.3 & & 0.66 & \\
\hline $21-5,137$ & -57.0 & 43.7 & 4.5 & 0.34 & 5 \\
\hline $139^{*}$ & -60.2 & 76.6 & & 0.23 & \\
\hline $22-1,0 *$ & -55.1 & 122.3 & & 0.38 & \\
\hline $2 *$ & -56.9 & 116.3 & & 0.35 & \\
\hline $4 *$ & -58.7 & 125.2 & & 0.33 & \\
\hline $6^{*}$ & -60.6 & 120.4 & & 0.36 & \\
\hline 8* & -59.9 & 123.2 & & 0.29 & \\
\hline $22-3,0 *^{\mathrm{a}}$ & -39.5 & 31.7 & & 0.17 & \\
\hline $2 *$ & -63.8 & 34.5 & & 0.39 & \\
\hline $4 *$ & -58.3 & 50.1 & & 0.37 & \\
\hline $23-1,144 *$ & -48.6 & 137.1 & & 0.32 & \\
\hline $146^{*}$ & -62.2 & 135.4 & & 0.29 & \\
\hline $148^{*}$ & -61.8 & 109.0 & & 0.29 & \\
\hline $23-2,103 *$ & 67.7 & 27.0 & & 0.31 & \\
\hline $105^{*}$ & 59.9 & 30.5 & & 0.30 & \\
\hline $107 *$ & 64.1 & 25.0 & & 0.30 & \\
\hline $111^{* a}$ & 46.0 & 320.8 & & 0.15 & \\
\hline $23-3,102 *$ & -63.3 & 138.0 & & 0.44 & \\
\hline $104^{*}$ & -67.6 & 153.2 & & 0.48 & \\
\hline $106^{*}$ & -55.3 & 143.3 & & 0.36 & \\
\hline $23-4,84 *$ & -56.9 & 85.4 & & 0.31 & \\
\hline $86 *$ & -69.1 & 83.2 & & 0.38 & \\
\hline $88 *$ & -61.6 & 96.0 & & 0.35 & \\
\hline $23-5,61^{*}$ & -63.1 & 140.7 & & 0.31 & \\
\hline $63^{*}$ & -60.7 & 145.4 & & 0.33 & \\
\hline $65^{*}$ & -53.6 & 138.3 & & 0.35 & \\
\hline $67 *$ & -66.7 & 154.1 & & 0.39 & \\
\hline $69 *$ & -63.3 & 133.3 & & 0.41 & \\
\hline $23-5,140^{*}$ & -66.5 & 283.4 & & 0.38 & \\
\hline $142^{*}$ & -68.6 & 293.0 & & 0.32 & \\
\hline $144 *$ & -66.6 & 267.0 & & 0.36 & \\
\hline $24-1,17$ & -50.2 & 82.4 & 4.4 & 0.53 & 6 \\
\hline 19 & -55.3 & 66.7 & 1.2 & 0.47 & 17 \\
\hline $24-2,97$ & -55.9 & 124.5 & 0.2 & 0.49 & 10 \\
\hline 99 & -62.5 & 127.2 & 0.7 & 0.43 & 14 \\
\hline
\end{tabular}

TABLE 1 - Continued

\begin{tabular}{|c|c|c|c|c|c|}
\hline $\begin{array}{l}\text { Core-Section } \\
\text { Interval }(\mathrm{cm})\end{array}$ & $\begin{array}{l}\text { Incl. } \\
(\circ)\end{array}$ & $\begin{array}{l}\text { Decl. } \\
(\circ)\end{array}$ & A & $\begin{array}{c}\begin{array}{l}\text { Intensity } \\
\times 10^{-6} \frac{\mathrm{emu}}{\mathrm{cc}}\end{array}\end{array}$ & $\begin{array}{l}\mathrm{MM} \\
(\%)\end{array}$ \\
\hline $24-3,146$ & -34.9 & 88.4 & 22.7 & 0.19 & 43 \\
\hline 148 & -49.0 & 73.4 & 14.0 & 0.18 & 14 \\
\hline $24-5,26$ & -77.7 & 232.6 & 6.6 & 0.25 & 27 \\
\hline 28 & -55.8 & 289.5 & 1.7 & 0.29 & 23 \\
\hline $24-6,110$ & -37.5 & 117.6 & 1.4 & 0.18 & 34 \\
\hline 112 & -43.6 & 121.4 & 0.3 & 0.19 & 23 \\
\hline 114 & -39.3 & 97.4 & 4.8 & 0.18 & 35 \\
\hline $25-1,135$ & -75.0 & 244.5 & 2.0 & 0.34 & 12 \\
\hline 137 & -69.6 & 236.8 & 0.1 & 0.33 & 15 \\
\hline 139 & -71.8 & 249.2 & 3.9 & 0.32 & 14 \\
\hline $25-2,119$ & -67.5 & 32.9 & 5.2 & 0.32 & 10 \\
\hline 121 & -63.9 & 8.4 & 5.1 & 0.27 & 13 \\
\hline $25-4,111$ & -56.4 & 95.3 & 1.6 & 0.26 & 24 \\
\hline 113 & -37.4 & 110.0 & 0.5 & 0.24 & 24 \\
\hline 115 & -35.5 & 177.2 & 20.7 & 0.21 & 38 \\
\hline $26-1,124$ & -42.0 & 91.3 & 0.5 & 0.28 & 21 \\
\hline 126 & -45.4 & 101.0 & 5.9 & 0.30 & 9 \\
\hline $26-2,141$ & -19.5 & 17.8 & 6.5 & 0.87 & 53 \\
\hline 143 & -75.6 & 193.6 & 7.3 & 0.19 & 22 \\
\hline 145 & -20.3 & 201.9 & 11.4 & 0.98 & 42 \\
\hline $26-4,144$ & -77.9 & 233.7 & 6.4 & 0.22 & 28 \\
\hline 146 & -81.2 & 235.6 & 1.3 & 0.28 & 17 \\
\hline 148 & -38.6 & 194.2 & 20.5 & 0.17 & 37 \\
\hline $26-5,27$ & -41.4 & 117.7 & 11.3 & 0.24 & 32 \\
\hline 29 & -42.5 & 92.3 & 0.3 & 0.29 & 15 \\
\hline 31 & -60.2 & 72.6 & 4.4 & 0.32 & 16 \\
\hline $28-1,57$ & -52.1 & 77.7 & 2.3 & 0.41 & 4 \\
\hline 59 & -58.5 & 85.7 & 2.3 & 0.46 & 12 \\
\hline 61 & -53.7 & 80.9 & 0.3 & 0.44 & 26 \\
\hline 63 & -58.4 & 101.5 & 2.4 & 0.46 & 12 \\
\hline 65 & -54.8 & 82.5 & 2.2 & 0.43 & 14 \\
\hline 67 & -47.5 & 84.9 & 1.8 & 0.38 & 21 \\
\hline $28-2,34$ & -66.2 & 48.9 & 3.2 & 0.39 & 23 \\
\hline 36 & -48.3 & 96.4 & 4.7 & 0.41 & 11 \\
\hline 38 & -51.1 & 86.4 & 7.9 & 0.35 & 11 \\
\hline $28-3,4$ & -83.3 & 68.0 & 2.6 & 0.27 & 16 \\
\hline 6 & -59.7 & 35.2 & 3.7 & 0.56 & 16 \\
\hline 8 & -62.8 & 11.7 & 5.6 & 0.31 & 16 \\
\hline 10 & -62.1 & 8.9 & 6.1 & 0.36 & 26 \\
\hline $28-3,113$ & -49.5 & 87.7 & 0.5 & 0.28 & 11 \\
\hline 115 & -34.6 & 92.4 & 0.2 & 0.23 & 23 \\
\hline $29-2,61$ & -69.4 & 167.9 & 6.8 & 0.20 & 24 \\
\hline 63 & -72.8 & 211.6 & 8.4 & 0.21 & 30 \\
\hline 99 & 57.4 & 13.1 & 3.3 & 0.23 & 24 \\
\hline 101 & 60.4 & 26.5 & 4.2 & 0.19 & 34 \\
\hline $29-4,37$ & -15.8 & 90.7 & 1.9 & 0.28 & 44 \\
\hline $29-4,91$ & -40.3 & 175.2 & 5.3 & 0.22 & 28 \\
\hline 93 & -56.6 & 153.3 & 8.1 & 0.20 & 18 \\
\hline
\end{tabular}

Note: Asterisk denotes Schonstedt magnetometer and ac demagnetization apparatus at MIT used.

${ }^{\mathrm{a}}$ Very unstable direction of magnetization.

1969; Pechersky and Khramov, 1973) or dominantly normal polarity with a single reversal (Pechersky and Khramov, 1973). The short middle to lower Albian reversal observed at Site 260 thus may correlate with the reversal assigned by Pechersky and Khramov (1973) to the Albian-Aptian boundary.

All samples at Site 261 are normally polarized. Cores 19-23 are Aptian in age; no reversed polarities have yet been reported from rocks of Aptian age. Cores 31 and 32 are lowest Cretaceous and uppermost Jurassic in age. 
TABLE 2

ac Demagnetization Results

\begin{tabular}{lccccc}
\hline $\begin{array}{c}\text { a.c. } \\
\text { Field } \\
(\text { oe })\end{array}$ & $\begin{array}{c}\text { Incl. } \\
(\circ)\end{array}$ & $\begin{array}{c}\text { Decl. } \\
(\circ)\end{array}$ & A & $\begin{array}{c}\text { Intensity } \\
\times 10^{-6} \text { emu } \\
\text { Site } 260, \text { Sample }\end{array}$ & $\begin{array}{c}\text { MM } \\
(\%)\end{array}$ \\
\hline \multicolumn{7}{l}{ S6-9 } \\
NRM & -56.7 & 270.3 & 0.3 & 23.7 & 12 \\
50 & -55.2 & 269.5 & 0.4 & 23.2 & 11 \\
200 & -50.2 & 267.7 & 0.7 & 17.0 & 12 \\
400 & -31.8 & 269.2 & 0.1 & 7.8 & 13
\end{tabular}

Site 263, Sample 18-4-34

$\begin{array}{cccccc}\text { NRM } & -48.6 & 317.9 & 3.4 & 0.64 & 11 \\ 25 & -45.5 & 317.8 & 2.7 & 0.58 & 11 \\ 50 & -43.3 & 320.3 & 1.3 & 0.54 & 15 \\ 75 & -41.9 & 319.7 & 0.0 & 0.50 & 17 \\ 100 & -42.7 & 316.2 & 1.4 & 0.46 & 11 \\ 150 & -32.6 & 325.8 & 0.1 & 0.38 & 17 \\ 200 & -32.0 & 328.3 & 0.3 & 0.35 & 21\end{array}$

Site 263, Sample 28-1-63

$\begin{array}{crrrrr}\text { NRM } & -69.4 & 118.6 & 2.7 & 0.50 & 14 \\ 25 & -55.5 & 93.9 & 4.7 & 0.50 & 9 \\ 50 & -58.4 & 101.5 & 2.4 & 0.56 & 12 \\ 75 & -59.2 & 99.8 & 3.4 & 0.41 & 11 \\ 100 & -54.5 & 106.3 & 2.9 & 0.38 & 17 \\ 150 & -50.6 & 102.0 & 1.9 & 0.30 & 24 \\ 200 & -47.6 & 96.1 & 6.5 & 0.26 & 22\end{array}$

Site 260, Sample 15-3-104

\begin{tabular}{cccccc} 
NRM & -47.7 & 178.6 & 0.2 & 1.29 & 13 \\
25 & -49.6 & 179.1 & 0.5 & 1.29 & 14 \\
50 & -51.3 & 178.2 & 0.3 & 1.25 & 14 \\
75 & -53.0 & 177.7 & 0.4 & 1.23 & 15 \\
100 & -53.1 & 177.5 & 0.2 & 1.19 & 14 \\
150 & -52.0 & 178.6 & 0.1 & 1.13 & 14 \\
200 & -51.3 & 176.3 & 1.3 & 1.05 & 15 \\
250 & -52.4 & 177.6 & 1.6 & 0.95 & 16 \\
300 & -49.4 & 178.2 & 1.5 & 0.92 & 14 \\
400 & -50.3 & 180.7 & 1.8 & 0.83 & 11 \\
\hline
\end{tabular}

Magnetic anomalies suggest frequent reversals throughout this time interval (Larson and Pitman, 1972). Because of the limited sample coverage of this interval at Site 261, the observation of only normal polarity probably is not incompatible with magnetic anomaly evidence.

Although paleontologically the sediments sampled at Site 263 are known to be lower Cretaceous, discrepancies between zonal assignments based on various taxa make the zones uncertain. The reversal stratigraphy at Site 263 (Figure 5) seems most consistent with placing the lower boundary of the long interval of normal polarity (and thus a Barremian age) in Core 20. The three reversals in Cores 20-23 are inconsistent with an Albian or Aptian age for this interval based on our limited present knowledge of Albian and Aptian reversal stratigraphy.

\section{PALEOLATITUDES}

Sclater and Cox (1970) showed that paleomagnetic inclinations of sediments at DSDP Site 10 in the North Atlantic were consistent with expected inclinations at the site based on North American paleomagnetic data. Paleomagnetic inclinations of Cretaceous sediments at Site 66 (Sclater and Jarrard, 1971) in the Central Pacific were consistent with Cretaceous virtual geomagnetic poles (VGPs) from seamounts (Francheteau et al., 1970) and with a paleolatitude from the Great Magnetic Bight (Vine, 1968). However, these three results indicate several degrees more northward motion of the Pacific plate since the Cretaceous than is indicated by inclinations at the Central Pacific DSDP Site 167 (Jarrard, 1973). Paleomagnetic inclinations of Cretaceous and Tertiary sediments at DSDP Site 212 in the Wharton Basin (not far from the three sites of the present study) are consistent with Australian paleomagnetic data. Paleolatitudes of basalt cores from DSDP Sites 260 and 261 are consistent with Jurassic paleomagnetic results from Australia, but a paleolatitude from DSDP Site 259 basalt cores is not consistent (McElhinney, this volume). Thus, it is of interest to examine paleolatitudes from Site 260,261 , and 263 sediment inclinations.

Median inclinations and 95\% confidence limits of the median for each site, along with resulting paleolatitudes (from $\tan$ [inclination] $=2$ tan [paleolatitude] were calculated from reliable $\mathrm{A}<5.0) 50$ oe paleomagnetic measurements (Table 3). Figure 6 shows paleocolatitudes (VGP loci) based on inclinations at Sites 260, 261, and 263 (UCSB measurements) and Site 263 (MIT measurements), compared with a Site 212 Cretaceous paleocolatitude (Jarrard and Sclater, 1974) and with Australian VGPs from the approximately 39-m.y. old Mt. Dromedary igneous complex (Robertson, 1963) and the approximately 104-m.y. old Cygnet alkaline complex (Robertson and Hastie, 1962). It is apparent that all paleocolatitudes are significantly different from the spin axis and that they intersect in the general region of the Australian VGPs. Thus, the paleomagnetism of Leg 27 sediments is consistent with the hypotheses of stable detrital remanent magnetization and of no relative motion of Sites 212, 260,261, 263, and Australia since the middle Cretaceous.

\section{ACKNOWLEDGMENTS}

I thank Ken Green for measuring many of the samples. Bruce Luyendyk and Robert Cockerham at the University of California at Santa Barbara made this project possible through their encouragement, assistance, and the use of their Schonstedt magnetometer. I thank John Sclater, John Veevers, and James Heirtzler for their interest in this project. This work was supported by National Science Foundation Grant GA-36589.

\section{REFERENCES}

Doell, R. R. and Cox, A., 1965. Measurement of the remanent magnetization of igneous rocks: U.S. Geol. Surv. Bull. 1203-A, p. 1-32.

Francheteau, J., Harrison, C. G. A., Sclater, J. G., and Richards, M. L., 1970. Magnetization of Pacific sea- 
TABLE 3

Paleolatitudes from Core Inclinations

\begin{tabular}{lccccc}
\hline Site & $\begin{array}{c}\text { Number } \\
\text { of Samples }\end{array}$ & $\begin{array}{c}\text { Median } \\
\text { Inclination }\end{array}$ & $\begin{array}{c}95 \% \text { Confidence } \\
\text { Limits of } \\
\text { the Median }\end{array}$ & Paleolatitude & $\begin{array}{c}\text { 95\% Confidence } \\
\text { Limits }\end{array}$ \\
\hline 260 & 68 & 44.5 & $39.4-46.9$ & 26.2 & $22.3-28.1$ \\
261 & 30 & 51.6 & $48.7-55.7$ & 32.2 & $29.6-36.2$ \\
$263^{\mathrm{a}}$ & 73 & 56.6 & $54.9-59.2$ & 37.2 & $35.4-40.0$ \\
$263^{\mathrm{b}}$ & 38 & 63.2 & $60.6-64.8$ & 44.7 & $41.6-46.7$ \\
\hline
\end{tabular}

a UCSB.

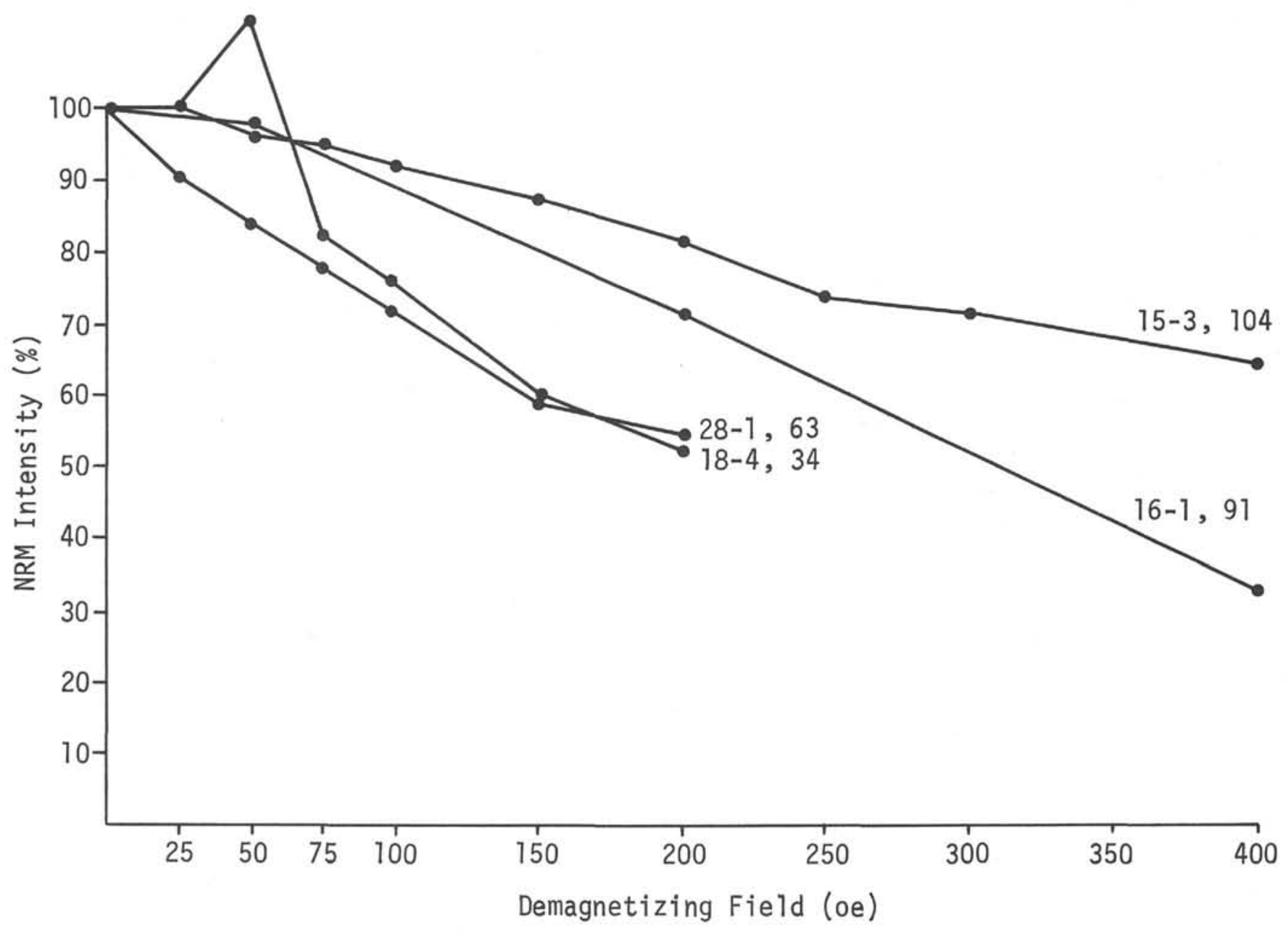

Figure 1. Cumulative demagnetization curves for four samples from Site 263.

mounts: a preliminary polar curve for the northeastern Pacific: J. Geophys. Res., v. 75, p. 2035.

Helsley, C. E. and Steiner, M. B., 1969. Evidence for long intervals of normal polarity during the Cretaceous period: Earth Planet. Sci. Lett., v. 5, p. 325.

Irving, E. and Covillard, R. W., 1973. Cretaceous normal polarity interval: Nature Phys. Sci., v. 244, p. 10.

Jarrard, R. D., 1973. Paleomagnetism of Leg 17 sediment cores. In Winterer, E. L., Ewing, J. L., et al., Initial Reports of the Deep Sea Drilling Project, Volume 17: Washington (U.S. Government Printing Office), p. 365.

Jarrard, R. D. and Sclater, J. G., 1974 . Preliminary paleomagnetic results, Leg 22. In von der Borsch, C., Sclater, J. G., et al., Initial Reports of the Deep Sea Drilling Project,
Volume 22:Washington (U.S. Government Printing Office).

Larson, R. L. and Pitman, W. C., III, 1972. Worldwide correlation of Mesozoic magnetic anomalies and its implications: Geol. Soc. Am. Bull., v. 83, p. 3645.

Pechersky, D. M. and Khramov, A. N., 1973. Mesozoic paleomagnetic scale of the USSR: Nature, v. 244, p. 499.

Robertson, W. A., 1963. The paleomagnetism of some Mesozoic intrusives and tuffs from eastern Australia: J. Geophys. Res., v. 68, p. 2299.

Robertson, W. A. and Hastie, L., 1962. A paleomagnetic study of the Cygnet alkaline complex of Tasmania: J. Geol. Soc. Australia, v. 8 , p. 259.

Sclater, J. G. and Cox, A., 1970. Paleolatitudes from JOIDES deep sea sediment cores: Nature, v. 226, p. 934. 

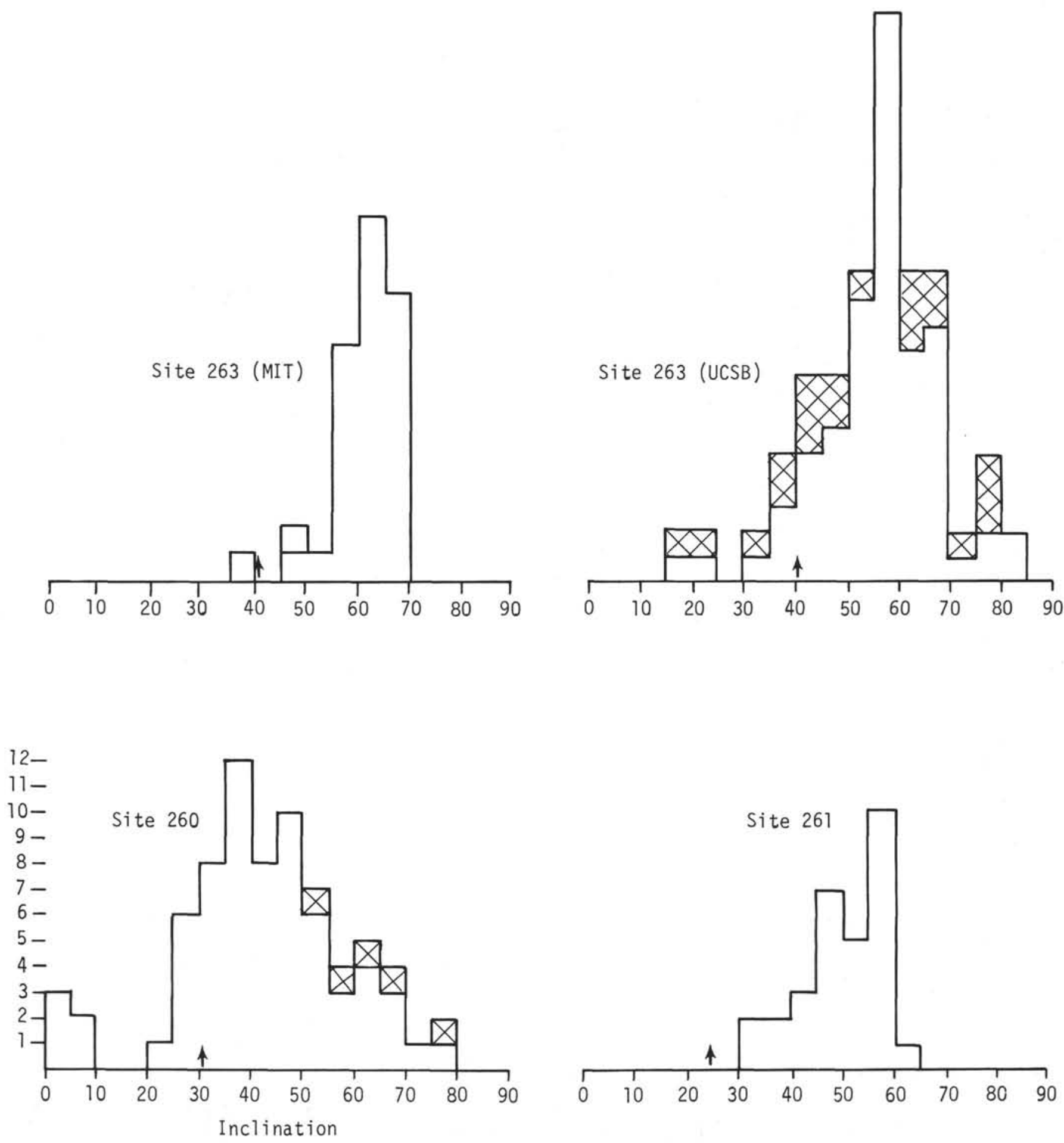

Figure 2. Histograms of absolute values of inclinations after 50-oe demagnetization. Arrows indicate inclination of axial geocentric dipole field for present latitudes of sites. Crosses indicate samples with poorly defined direction of magnetization $(A \geqslant 5)$

Sclater, J. G. and Jarrard, R. D., 1971. Preliminary paleomagnetic results, Leg 7. In Winterer et al., Initial Reports of the Deep Sea Drilling Project, Volume 7: Wahington (U.S. Government Printing Office), p. 1227.

Sclater, J. G., Jarrard, R. D., McGowran, B., and Gartner, S., in press. Comparison of the magnetic and biostratigraphic time scales since the Late Cretaceous. In Von der Borsch,
C., Sclater, J. G., et al., Initial Reports of the Deep Sea Drilling Project, Volume 22: Washington (U.S. Government Printing Office).

Vine, F. J., 1968. Paleomagnetic evidence for the northward movement of the North Pacific Basin during the past 100 m.y. (abstract): Am. Geophys. Union Trans., v. 49, p. 156. 

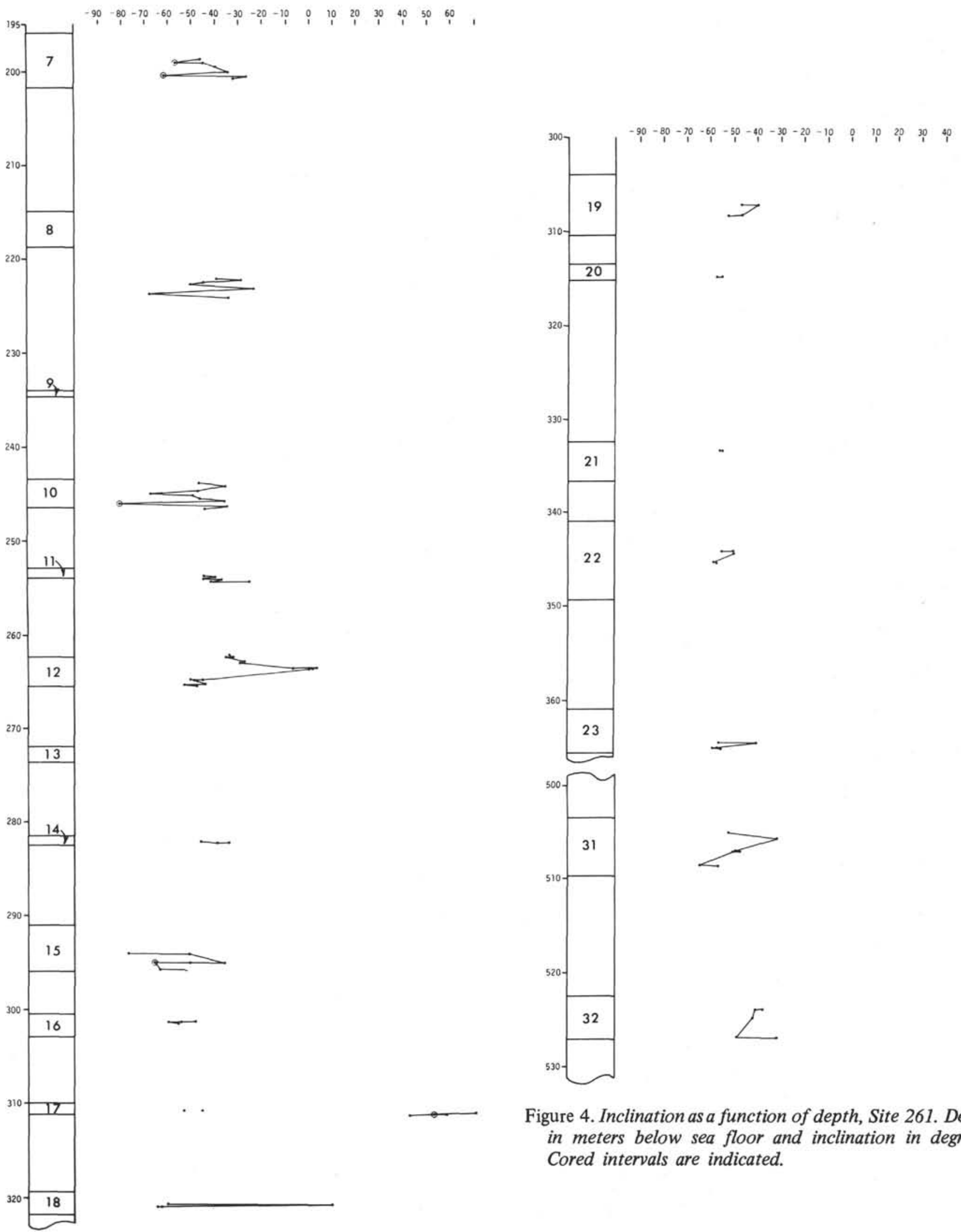

Figure 4. Inclination as a function of depth, Site 261. Depth in meters below sea floor and inclination in degrees. Cored intervals are indicated.

Figure 3. Inclination as a function of depth, Site 260. Depth in meters below sea floor and inclination in degrees. Cored intervals are indicated. 
R. D. JARRARD

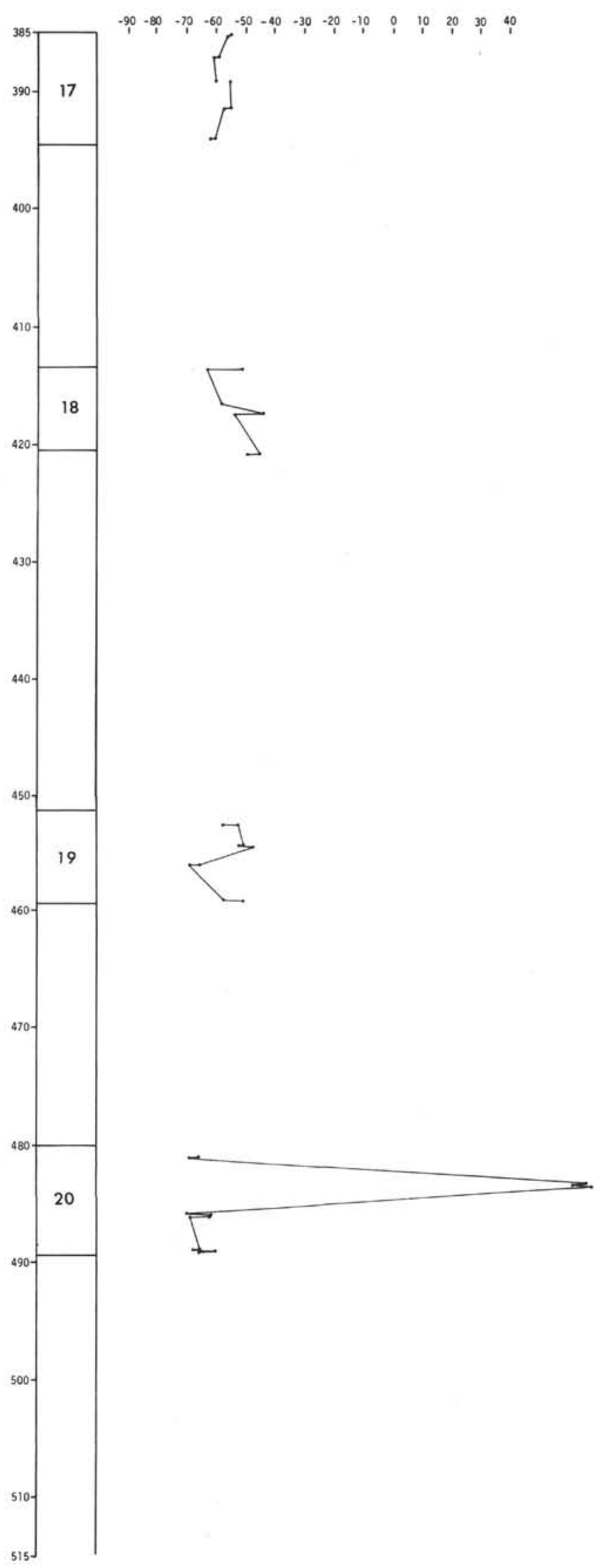

Figure 5. Inclination as a function of depth, Site 263. Depth in meters below sea floor and inclination in degrees. Cored intervals are indicated.

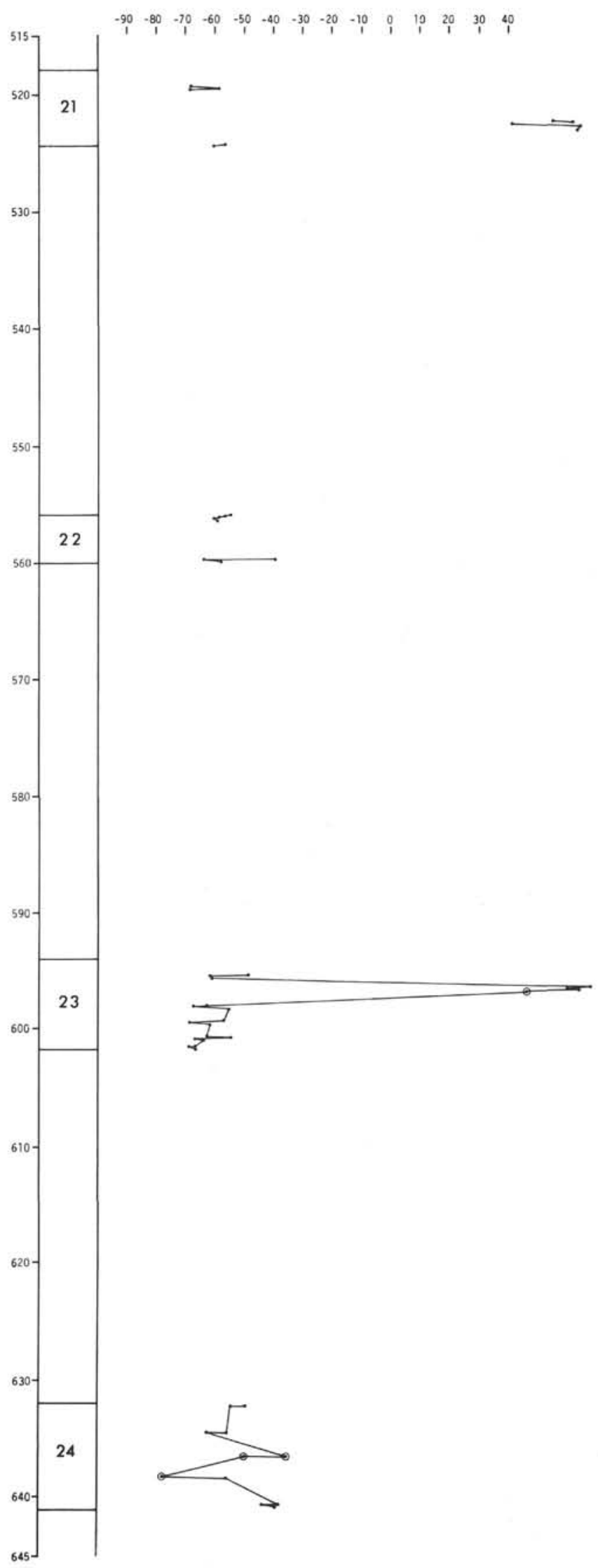

Figure 5. (Continued).

422 

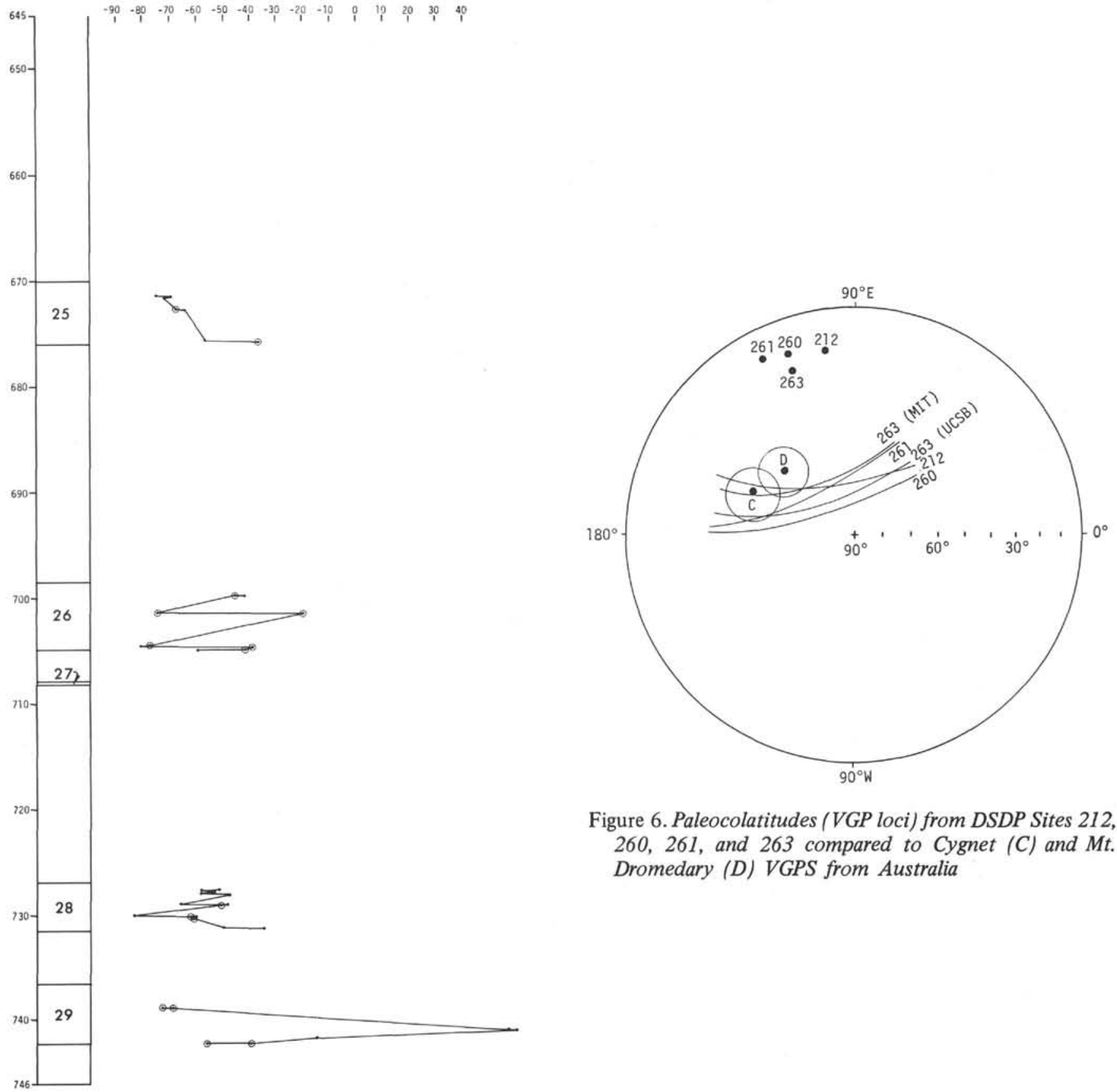

Figure 6. Paleocolatitudes (VGP loci) from DSDP Sites 212, 260, 261, and 263 compared to Cygnet (C) and Mt. Dromedary (D) VGPS from Australia

Figure 5. (Continued). 